\title{
Reovirus infection induces apoptosis of TRAIL-resistant gastric cancer cells by down-regulation of Akt activation
}

\author{
IL-RAE CHO $^{1}$, SANG SEOK KOH ${ }^{3}$, HYE-JIN MIN ${ }^{3}$, EUN-HEE PARK ${ }^{1}$, \\ RATAKORN SRISUTTEE ${ }^{1}$, BYUNG HAK JHUN ${ }^{2}$, CHI DUK KANG ${ }^{4}$, MANBOK KIM $^{5}$, \\ RANDAL N. JOHNSTON ${ }^{6}$ and YOUNG-HWA CHUNG ${ }^{1}$
}

\begin{abstract}
Departments of ${ }^{1}$ Cogno-Mechatronics Engineering, BK21 Nanofusion Technology Team, ${ }^{2}$ Nanomedical Engineering, Pusan National University, Busan 609-735; ${ }^{3}$ Therapeutic Antibody Research Center, Korea Research Institute of Bioscience and Biotechnology, Daejeon 305-333; ${ }^{4}$ Department of Biochemistry, Pusan National University, Busan 602-739; ${ }^{5}$ Institute for Cancer Research, College of Medicine, Yonsei University, Seoul 120-740, Korea; ${ }^{6}$ Department of Biochemistry and Molecular Biology, University of Calgary, Calgary, Alberta, T2N 4N1, Canada
\end{abstract}

Received November 2, 2009; Accepted January 5, 2010

DOI: 10.3892/ijo_00000583

\begin{abstract}
The tumor necrosis factor (TNF)-related apoptosisinducing ligand (TRAIL) has been used to treat a variety of cancer cells. However, since some gastric cancer cells are resistant to TRAIL, we explored whether reovirus induces cytolysis in TRAIL-resistant gastric cancer cells. We found that TRAIL-resistant SNU-216 gastric cancer cells were susceptible to apoptosis by reovirus infection. Furthermore, co-treatment with reovirus and TRAIL accelerated apoptosis of SNU-216 cells by down-regulation of Akt activation as assessed by a very low activation of Akt in TRAIL-sensitive SNU-668 gastric cancer cells. Inhibition of Akt signaling with wortmannin or suppression of Akt expression with sh-Akt lentivirus promoted reovirusmediated apoptosis of SNU-216 gastric cancer cells. Reovirus infection also down-regulates the activation of signaling molecules such as Ras and ERK involved in cell proliferation and survival but not the activation of p38 MAPK involved in cellular stress. In addition, the cotreatment with reovirus and TRAIL resulted in cleavage of caspase- 8 , caspase- 9 and Bid, leading to a decrease in the mitochondrial membrane potential, indicating that reovirus may utilize the mitochondrial intrinsic apoptotic pathway in TRAIL-resistant SNU-216 gastric cancer cells. Accordingly, we first demonstrate that reovirus infection down-regulates Akt
\end{abstract}

Correspondence to: Dr Young-Hwa Chung, Department of CognoMechatronics Engineering, BK21 Nanofusion Technology Team, Pusan National University, Busan 609-735, Korea

E-mail: younghc@pusan.ac.kr

Abbreviations: TRAIL, tumor necrosis factor-related apoptosisinducing ligand; DR, death receptor; $\operatorname{DiOC}_{6}(3), 3,3$ '-dihexyloxacarbocyanine; sh RNA, short hairpin RNA

Key words: TRAIL, gastric cancer, reovirus, Akt activation, leading to apoptosis of TRAIL-resistant gastric cancer cells.

\section{Introduction}

TNF-related apoptosis-inducing ligand (TRAIL), which belongs to the family of TNF, induces apoptosis in a wide variety of tumor cells in vitro and in vivo but does not cause toxicity in a majority of normal cells $(1,2)$. Thus, TRAIL has been suggested as a novel anti-cancer therapeutic drug. The TRAIL-mediated apoptotic signal is transduced through the cell surface death receptor (DR)s such as DR4/TRAIL-R1 and DR5/TRAIL-R2 $(3,4)$. Detailed studies have shown that TRAIL triggers apoptosis by recruiting the initiator procaspase- 8 through the adaptor protein FADD. Caspase- 8 can directly activate downstream effector caspases including procaspase-3, -6 and -7 , or cleave Bid, which triggers mitochondrial damage $(5,6)$. However, different types of cancer cells appear to differ in their sensitivity to TRAIL treatment. Recent studies have reported that prostate and renal cancer cells are resistant to TRAIL treatment due to an up-regulation of Akt activity and enhanced FLIP expression $(7,8)$. In addition, it has been reported that the resistance of certain gastric cancer cells to TRAIL-induced apoptosis can be explained by the up-regulation of FLIPs by Akt, indicating that Akt is a crucial component in the regulation of TRAIL-induced apoptosis (9). In contrast, other studies failed to demonstrate a link between FLIP expression and TRAIL resistance using melanoma and Burkitt's lymphoma $(10,11)$.

The human reovirus is a ubiquitous, non-enveloped virus with 10 segments of double-stranded RNA (12). The virus infection is usually restricted to the upper respiratory and gastrointestinal tracts and is often asymptomatic (12). However, the reovirus shows dramatic cytolytic activity in certain types of transformed cells $(13,14)$. There is a strong body of evidence that Ras-transformed cells are preferentially susceptible to reovirus (type 3 Dearing strain) via inactivation of PKR (dsRNA-activated protein kinase) phosphorylation $(15,16)$. Accordingly, activation of the oncogenic Ras-signaling path- 
way enhances reoviral oncolytic targeting in various types of human cancers (17-19), although our recent study shows that other signaling pathways may also contribute to the susceptibility of these cancers to reoviral replication and oncolysis (20). In addition, our study first reports that reovirus infection induces down-regulation of hypoxia-inducible factor (HIF)- $1 \alpha$ independently of VHL and p53, suggesting that reovirus may be useful as a potential therapeutic agent against chemoresistant or radioresistant tumors that are hypoxic and show increased levels of HIF-1 $\alpha$ (21). It has also been reported that reoviral oncolysis was associated with the induction of apoptosis in various cancer types (22). Further investigation has revealed that TRAIL is involved in reovirus-induced apoptosis (23). Caspase-8 and JNK MAPK proteins also participate in apoptotic signaling following reoviral infection (24).

Gastric cancer is one of the most common malignancies of the gastrointestinal tract in East Asia, including Japan, Korea and other South Asia countries, and the second most common cause of cancer-related death in the world (25). Since cultured gastric cancer cell lines are useful tools in the study of cell biology and the development of new therapeutic modalities, several different gastric carcinoma cell lines have been established (26,27). Gastric cancer cell lines, SNU-216 and SNU-668, have been reported to possess a p53 mutation at exon 6 and a c-Ki-ras mutation (28), respectively. In addition, a subsequent study has shown that SNU-216 cells are resistant to TRAIL while SNU-668 cells are susceptible to TRAIL treatment (9).

Since other groups have not attempted the potential application of reovirus to a gastric cancer model until now, we initiated this study to explore whether the reovirus can efficiently act as an anti-cancer drug by exerting cytolysis in TRAIL-resistant SNU-216 gastric cancer cells. We report that the reovirus facilitated the induction of apoptosis in SNU-216 gastric cancer cells by down-regulation of Akt activation and rendered the SNU-216 cells susceptible to TRAIL, suggesting that there may a possibility for the potential application of reovirus in the treatment of human gastric cancer cells bearing active Akt.

\section{Materials and methods}

Cell cultures and virus amplification. SNU-216, SNU-668 gastric cancer cells (KTCC, Seoul, Korea), and murine L929 cells were cultured in DMEM supplemented with $10 \%$ FBS and $1 \%$ penicillin and streptomycin. The Dearing strain of reovirus serotype 3, purchased from the ATCC (Manassas, VA), was propagated in L929 cells. Reovirus was purified as described elsewhere (29) and the viral titer was measured in plaque forming units (PFU).

Reagents and antibodies. Wortmannin and TRAIL were purchased from Calbiochem (San Diego, CA) and R\&D Systems (Minneapolis, MN), respectively. For immunoblotting, anti-caspase-8, -caspase-9, -Bid, and - - -tubulin antibodies were obtained from Santa Cruz Biotech (Santa Cruz, CA). For detection of caspase- 3 or -7 activity, the PARP level was detected with immunoblotting using anti-PARP (Cell Signaling Biotech, Danvers, MA) ERK, p38 MAPK, Akt, and their phospho-specific antibodies were purchased from
Cell Signaling Biotech. Polyclonal anti-reovirus antibody was used for the detection of $\lambda, \mu$ and $\sigma$ reoviral proteins as described previously (30).

Western blot assay. Cells were harvested and lysed with lysis buffer [150 mM NaCl, 1\% NP-40, $50 \mathrm{mM}$ Tris-HCl $(\mathrm{pH}$ 7.5)] containing $0.1 \mathrm{mM} \mathrm{Na} \mathrm{VO}_{3}, 1 \mathrm{mM} \mathrm{NaF}$ and protease inhibitors (Sigma, St. Louis, MO). For immunoblotting, proteins from whole cell lysates were resolved by $10 \%$ or $15 \%$ SDS-PAGE and then transferred to nitrocellulose membranes. Primary antibodies were used at 1:1000 or 1:2000 dilutions, and secondary antibodies conjugated with horseradish peroxidase were used at 1:2000 dilutions in 5\% non-fat dry milk. After the final washing, nitrocellulose membranes were exposed for an enhanced chemiluminescence assay using the LAS 3000 (Fuji, Japan).

Active Ras capture assays. To capture GTP-bound Ras from cell lysates, a GST-Raf-RBD fusion protein was used as described elsewhere (22). The cells were lysed with lysis buffer and glutathione-agarose beads (Clontech, Mountain View, CA) were added to the supernatant and rocked on ice for $30 \mathrm{~min}$ to exclude non-specific binding. After centrifugation, the supernatant was harvested, followed by the addition of GST-Raf-RBD (Cytoskeleton). The solution was rocked on ice for $1 \mathrm{~h}$ followed by the addition of glutathione-agarose beads to capture active Ras, which binds to GST-Raf-RBD. After washing three times, the pellet was mixed with $40 \mu 1$ of $2 \mathrm{X}$ SDS loading buffer. To detect the captured Ras, immunoblotting was performed with anti-Ras antibody (Santa Cruz).

Infection of short hairpin(sh) RNA expression lentivirus for targeting Akt. To suppress expression of Akt, the shRNA expression lentiviral vectors for targeting Akt was constructed by inserting synthetic double strand oligonucleotides (for Akt: 5'-CGGAATTCCGGCCTGCCCTTCTACAACCAG GA ttcaagaga TCCTGGTTGTAGAAGGGCAGGTTTTTGA TATCTAGACA-3') into EcoRI-XbaI restriction enzyme sites of shLenti3.4G lentiviral vector and verified by nucleotide sequencing (Macrogen, Seoul, Korea). Lentivirus particles were produced as described elsewhere $(31,32)$ and concentrated to $10^{9}$ transduction unit by Macrogen. According to the manufacturer's recommendation, shRNA expression lentivirus for targeting Akt was infected (MOI=10) into SNU-216 gastric cancer cells and reovirus was subsequently treated $(\mathrm{MOI}=1)$ for $72 \mathrm{~h}$ before harvest.

Measurement of mitochondrial membrane potential. Retention of 3,3'-dihexyloxacarbocyanine, $\operatorname{DiOC}_{6}(3)$ was used as a measure of mitochondrial energization as described elsewhere (33). Cells were loaded with $40 \mathrm{nM} \mathrm{DiOC} 6$ (3) for $30 \mathrm{~min}$ followed by trypsinization. The concentration of retained $\mathrm{DiOC}_{6}(3)$ was determined on a fluorescence spectrometer (Victor 3, Perkin-Elmer, Waltham, MA) at an excitation and emission of $480 \mathrm{~nm}$ and $510 \mathrm{~nm}$, respectively.

\section{Results}

Reovirus infection causes SNU-216 gastric cancer cell susceptibility to TRAIL. TRAIL has been known to induce 
A

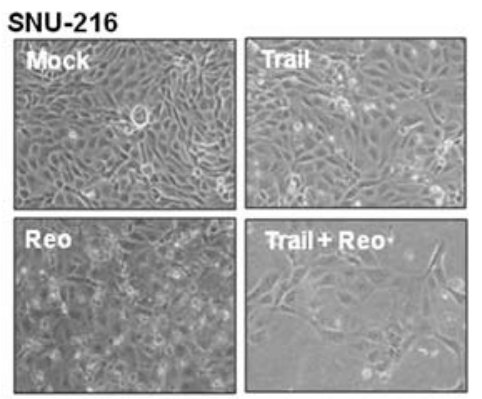

B

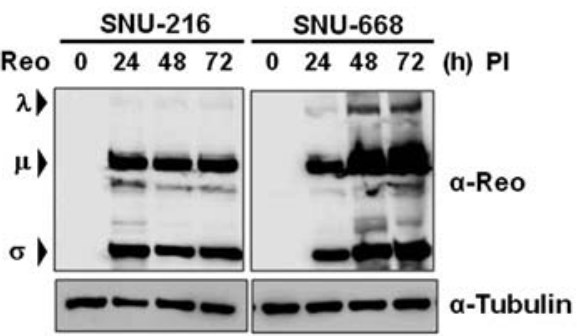

SNU-668

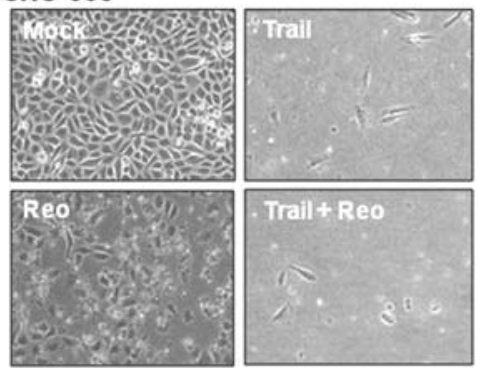

C

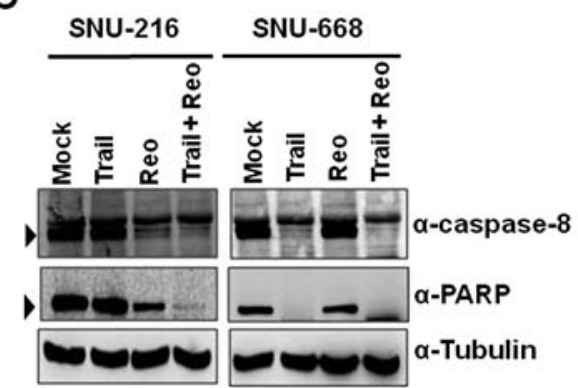

Figure 1. Reovirus infection enhances TRAIL sensitivity in SNU-216 gastric cancer cells. SNU-216 and SNU-668 cells were treated with TRAIL (50 ng/ml), reovirus (MOI=1), or TRAIL and reovirus for $72 \mathrm{~h}$. Both cell lines were observed by microscopy and images were acquired at $72 \mathrm{~h}$ post-treatment. Caspase- 8 , PARP and reoviral proteins were detected with immunoblotting using appropriate antibodies. Polyclonal anti-reovirus antibodies cross-reacts with $\lambda, \mu$ and $\sigma$ reoviral proteins. Arrow indicates a precursor form of caspase- 8 and uncleaved PARP.

apoptosis in a wide variety of tumor cells but not all tumor cell lines respond to TRAIL $(7,8)$. We confirmed that SNU216 gastric cancer cells are TRAIL-resistant while SNU-668 gastric cancer cells are susceptible to TRAIL as described previously (9) (Fig. 1A). Next, to examine whether TRAILresistant SNU-216 cells are susceptible to reovirus infection, the cell line was infected with reovirus and cell viability was examined during the infection. We found that SNU-216 cells started to display cytolysis at $48 \mathrm{~h}$ post-infection (data not shown) and clearly showed cell death at $72 \mathrm{~h}$ post-infection (Fig. 1A). SNU-668 cells also exhibited susceptibility to reovirus during the infection (Fig. 1A). When we examined reoviral propagation in both SNU-216 and SNU-668 cells, SNU-668 cells displayed slightly more reoviral propagation than SNU-216 (Fig. 1B), suggesting that the kinetics against viral infection are faster than with SNU-216 cells.

Furthermore, we examined whether the combined treatment using the reovirus and TRAIL could efficiently induce apoptosis in SNU-216 cells. SNU-216 and SNU-668 cells were subjected to treatment with TRAIL, reovirus, or reovirus together with TRAIL. In these experiments, we found that the reovirus infection more efficiently induced apoptosis in TRAIL-resistant SNU-216 cells in the presence of TRAIL compared to reovirus infection alone, suggesting that the reovirus sensitizes the TRAIL-resistant gastric cancer cells to apoptosis in the presence of TRAIL (Fig. 1A). In addition, reovirus infection resulted in activation of caspase- 8 and caspase-3 or -7 (because of cleavage of PARP) in SNU-216 cells (Fig. 1C) while activation of caspase-8 and cleavage of PARP did not occur during TRAIL treatment alone. However, the combined treatment accelerated reovirus-induced cleavage of pro-caspase 8 and PARP. Since SNU-668 cells were even susceptible to a lower concentration of TRAIL (20 ng/ml) (data not shown), TRAIL treatment resulted in a drastic increase of pro-caspase- 8 and PARP cleavage in SNU-668 cells. Of interest, SNU-668 gastric cancer cells did not show cleavage of pro-caspase-8 and PARP as seen in SNU-216 cells (Fig. 1C), indicating that SNU-668 gastric cancer cells may utilize a different apoptotic pathway that is taken by SNU-216 cells during reovirus infection. Taken together, these combined results indicate that reovirus infection renders the SNU-216 cells susceptible to TRAIL.

Reovirus infection decreases Akt activation in TRAIL-resistant $S N U-216$ cells. Since it has been suggested that TRAIL sensitivity in gastric cancer cells depends on PI3-kinase/Akt activation (9), we first examined Akt phosphorylation in both cell lines during reovirus infection using the antiphospho(Ser473)-Akt antibody. The phosphorylated Akt was considered as an activated Akt according to a previous study shown that Akt is activated by phospholipid binding and phosphorylation at Thr308 and at Ser473 (34). Surprisingly, we found that reovirus infection reduces phosphorylation of Akt in SNU-216 cells in a time-dependent manner (Fig. 2A), indicating that reovirus infection targets down-regulation of Akt activation. We did not observe any alternations in Akt phosphorylation for SNU-668 cells since almost no phosphorylation of Akt was detected in the uninfected SNU-668 cells. Furthermore, since we questioned how phosphorylation of Akt was down-regulated with reovirus infection, we examined whether activation of PTEN or Ras protein closely related to Akt signaling is affected by the infection. Phosphorylation of PTEN was not altered during reovirus infection (data not shown). However, abundance and activity of Ras protein was decreased during the infection (Fig. 2B). Accordingly, phosphorylation of ERK, a downstream 
A

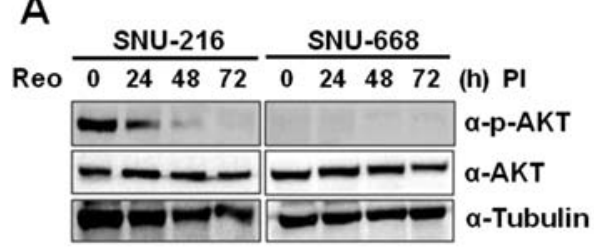

C

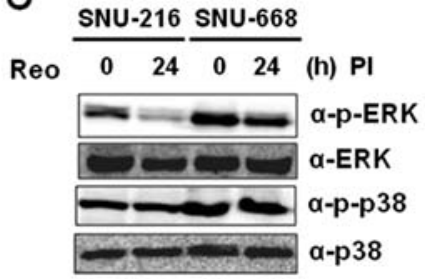

B

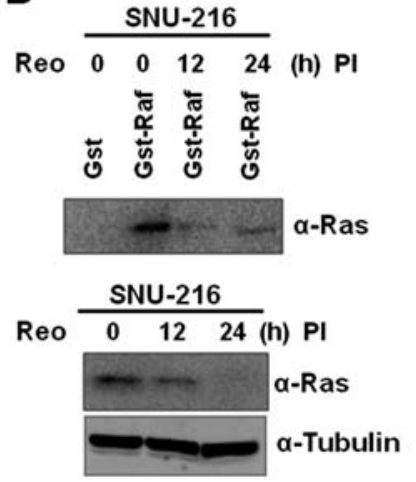

D

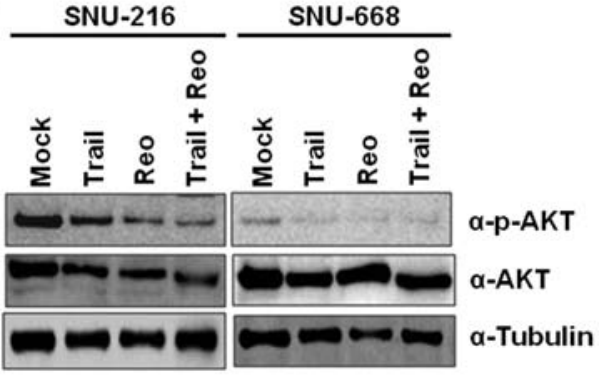

Figure 2. Reovirus infection down-regulates Akt phosphorylation in SNU-216 cells. (A and C) SNU-216 and SNU-668 cells were infected with reovirus $(\mathrm{MOI}=1)$ and harvested at 24, 48, and $72 \mathrm{~h}$ post-infection. Akt, ERK, p38 MAPK and their phospho-proteins were detected with immunoblotting using the corresponding antibody. Anti-phospho-Akt antibody; Ser473 of Akt, anti-phopsho ERK antibody; Thr202/Tyr204 of ERK, anti-p38 antibody; Thr180/Tyr182 of p38. (B) SNU-216 cells were infected with reovirus (MOI=10) and harvested at 12 and 24 h post-infection. GST-Raf-RBD protein was added into the cell lysate as a substrate of active Ras while GST was used for a control. Active Ras was pulled down with glutathione-agarose and the precipitated mixtures were separated on $12 \%$ SDS-PAGE and Ras protein was detected with anti-Ras antibody. (D) SNU-216 and SNU-668 cells were treated with TRAIL $(50 \mathrm{ng} / \mathrm{ml})$, reovirus (MOI=10), or TRAIL and reovirus for $24 \mathrm{~h}$. Proteins were analyzed with immunoblotting as described (A and C).

molecule in the Akt signaling pathway, was decreased during reovirus infection while phosphorylation of p38 MAPK was not altered (Fig. 2C). We also examined Akt phosphorylation in TRAIL-resistant SNU-216 cells treated with TRAIL alone, reovirus alone or reovirus and TRAIL. TRAIL treatment alone mildly reduced phosphorylation of Akt but reovirus infection significantly reduced phosphorylation of Akt (Fig. 2D). The combined treatment with reovirus and TRAIL also markedly inhibited Akt phosphorylation, supporting the notion that SNU-216 cells are more sensitive to apoptosis during co-treatment than during TRAIL or reovirus treatment alone (Fig. 2D).

Inhibition of Akt signaling sensitizes reovirus-induced apoptosis in TRAIL-resistant SNU-216 gastric cells. Since we have observed that co-treatment with reovirus and TRAIL almost completely reduces Akt phosphorylation in TRAILresistant SNU-216 gastric cancer cells (Fig. 2), we employed wortmannin, a PI-3 kinase inhibitor, to suppress PI-3 kinase/ Akt signaling. To determine whether co-treatment with reovirus and wortmannin can efficiently induce apoptosis in SNU-216 cells, SNU-216 cells were treated with TRAIL alone, wortmannin alone and both TRAIL and wortmannin. As shown in Fig. 3A, SNU-216 cells were strongly resistant to TRAIL treatment and only displayed a cell death of $\sim 10 \%$ at $72 \mathrm{~h}$ after wortmannin treatment compared to the mock treatment. However, co-treatment with TRAIL and wortmannin synergistically induced $65 \%$ cell death compared to the mock treatment. When we examined Akt phosphorylation in SNU-216 cells treated with TRAIL alone, wortmannin alone, or TRAIL and wortmannin, we found that co-treatment resulted in almost no Akt phosphorylation in SNU-216 cells although a slightly reduced phosphorylation of Akt was observed in the other treatments (Fig. 3B). This result suggests that down-regulation of Akt signaling by wortmannin enhances the sensitivity of SNU-216 gastric cancer cells to TRAIL-mediated apoptosis.

Next, to test our hypothesis, we examined whether cotreatment with reovirus and wortmannin accelerates apoptosis of SNU-216 cells by down-regulation of Akt activation. As shown in Fig. 3C, co-treatment with reovirus and wortmannin efficiently induced cell death of SNU-216 cells by up to 95\% compared to the mock treatment. Reovirus infection alone and wortmannin treatment alone induced $~ 55 \%$ and $10 \%$ cell death of SNU-216 cells, respectively, compared to the mock treatment. When we examined Akt phosphorylation in SNU-216 cells treated with reovirus alone, wortmannin alone, or reovirus and wortmannin at $24 \mathrm{~h}$ post-treatment, we found that co-treatment with wortmannin and reovirus results in almost no Akt phosphorylation in SNU-216 cells (Fig. 3D).

Directly to prove a crucial role of Akt in reovirus-induced apoptosis in SNU-216 gastric cancer cells, we employed shRNA expression lentivirus for targeting Akt (sh-Akt lentivirus) to suppress Akt expression. As shown in Fig. 4A, infection of sh-Akt lentivirus reduced expression of Akt while sh-Scramble lentivirus as a control did not. When SNU-216 cells were treated with the control lentivurs, sh-Akt lentivirus, reovirus, or sh-Akt lentivirus together with reovirus, cell viability and Akt phosphorylation were examined. We found that co-infection of reovirus and sh-Akt lentivirus drastically reduced $\sim 70 \%$ cell death compared to transduction of shScramble lentivirus while sh-Akt lentivirus infection alone did not induce cell death and reovirus infection mildly induced cell death (Fig. 4B). We also noted that the co-infection 

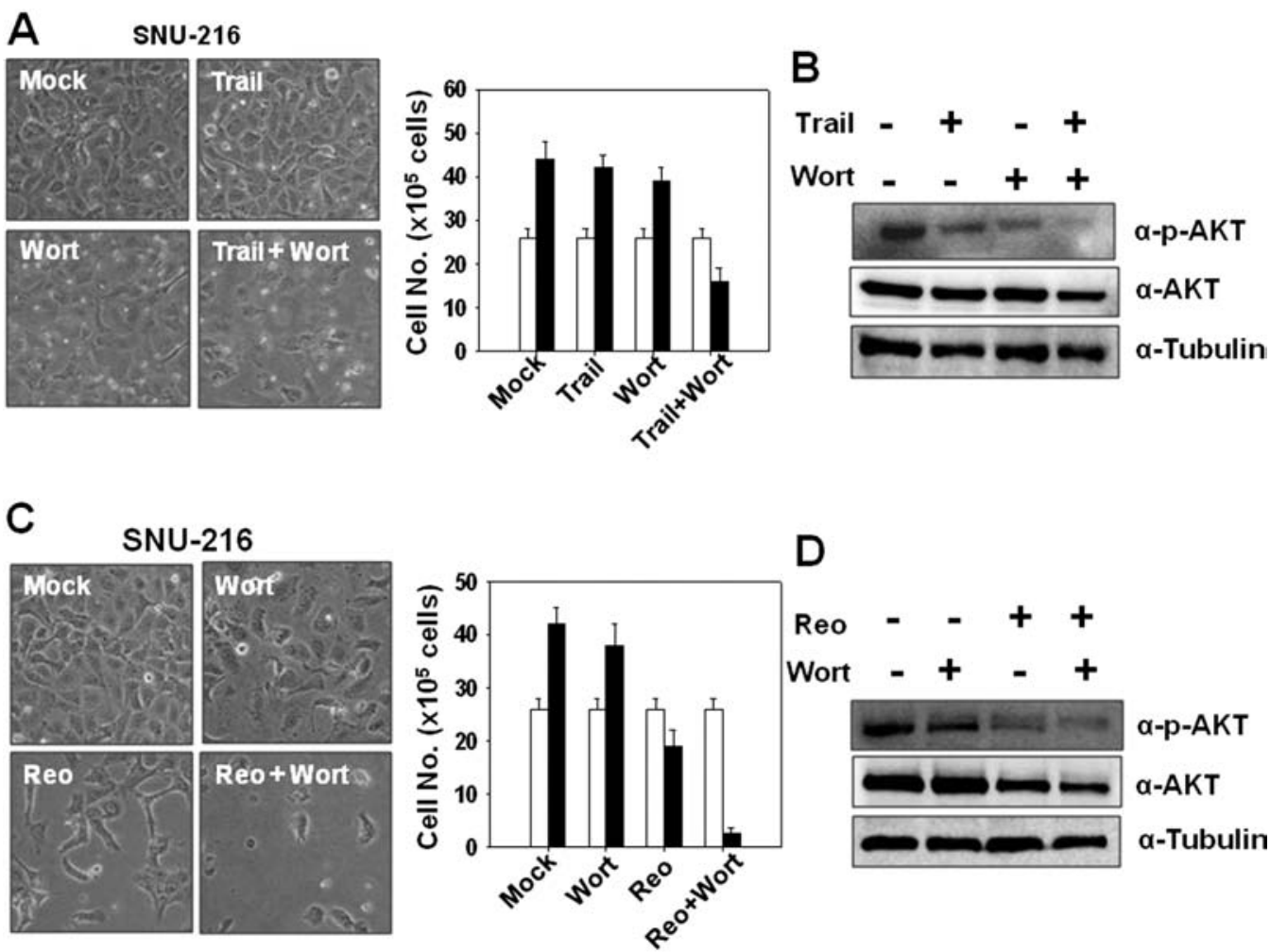

D

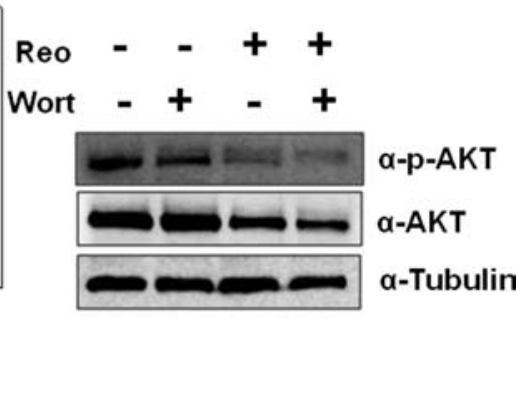

Figure 3. Inhibition of Akt signaling synergizes reovirus-mediated apoptosis in TRAIL-resistant SNU-216 cells. (A and C) SNU-216 cells were treated with TRAIL $(50 \mathrm{ng} / \mathrm{ml})$, wortmannin $(100 \mathrm{nM})$, or TRAIL plus wortmannin for $24 \mathrm{~h}$. SNU-216 cells were treated with reovirus (MOI=10), wortmannin or reovirus plus wortmannin for $24 \mathrm{~h}$. The cells were observed by microscopy and counted by trypan blue at $24 \mathrm{~h}$ post-infection. White bars indicate the cell number of inoculation at $0 \mathrm{~h}$ while black bars indicate the cell number treated with indicated agents at $24 \mathrm{~h}$. (B and D) The cells treated with indicated agents were harvested and cell lysates were prepared at $24 \mathrm{~h}$ post-treatment. Phosphor-Akt, and Akt were detected by immunoblotting with the appropriate antibody.

A

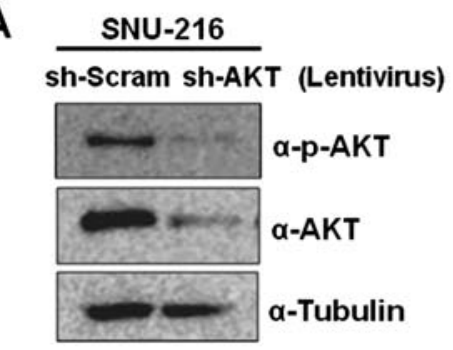

B

SNU-216
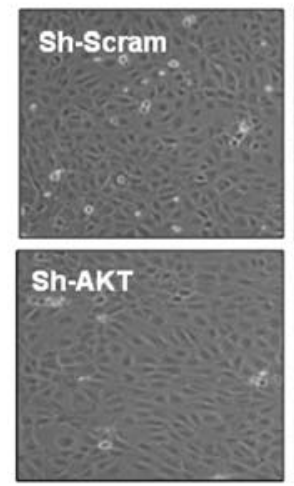

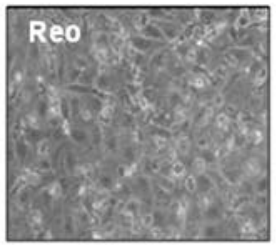

Sh-AKT+Reo:

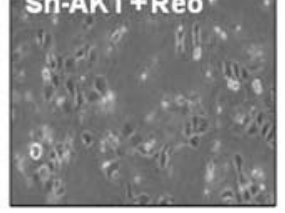

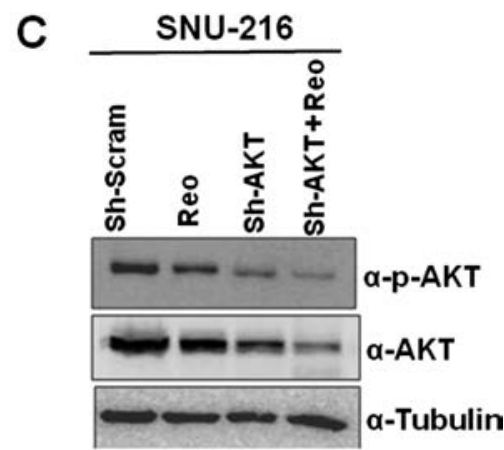

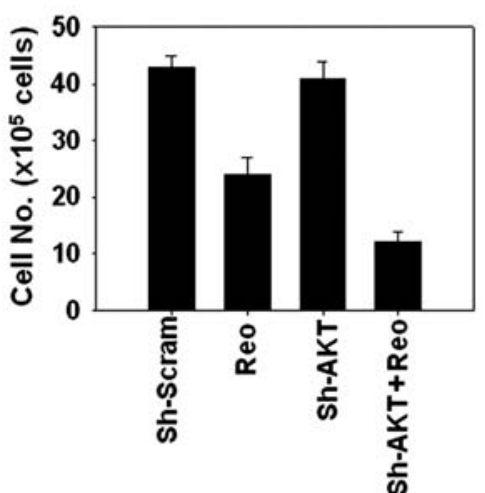

Figure 4. Suppression of Akt expression with sh-Akt lentivirus promotes reovirus-mediated apoptosis of SNU-216 cells. (A) SNU-216 cells were transduced with sh-Akt lentivirus (MOI=10) to reduce the expression of Akt for $48 \mathrm{~h}$ and sh-Scramble lentivirus (MOI=10) was used a negative control. (B and C) SNU-216 cells were treated with sh-Scramble lentivirus, sh-Akt lentivirus, reovirus (MOI=1), or sh-Akt lentivirus and reovirus for $72 \mathrm{~h}$. The treated cells were observed by microscopy and images were acquired before cell count using trypan blue at $72 \mathrm{~h}$ post-treatment. Phosphorylation level of Akt was examined with immunoblotting using anti-phospho-Akt antibody. 

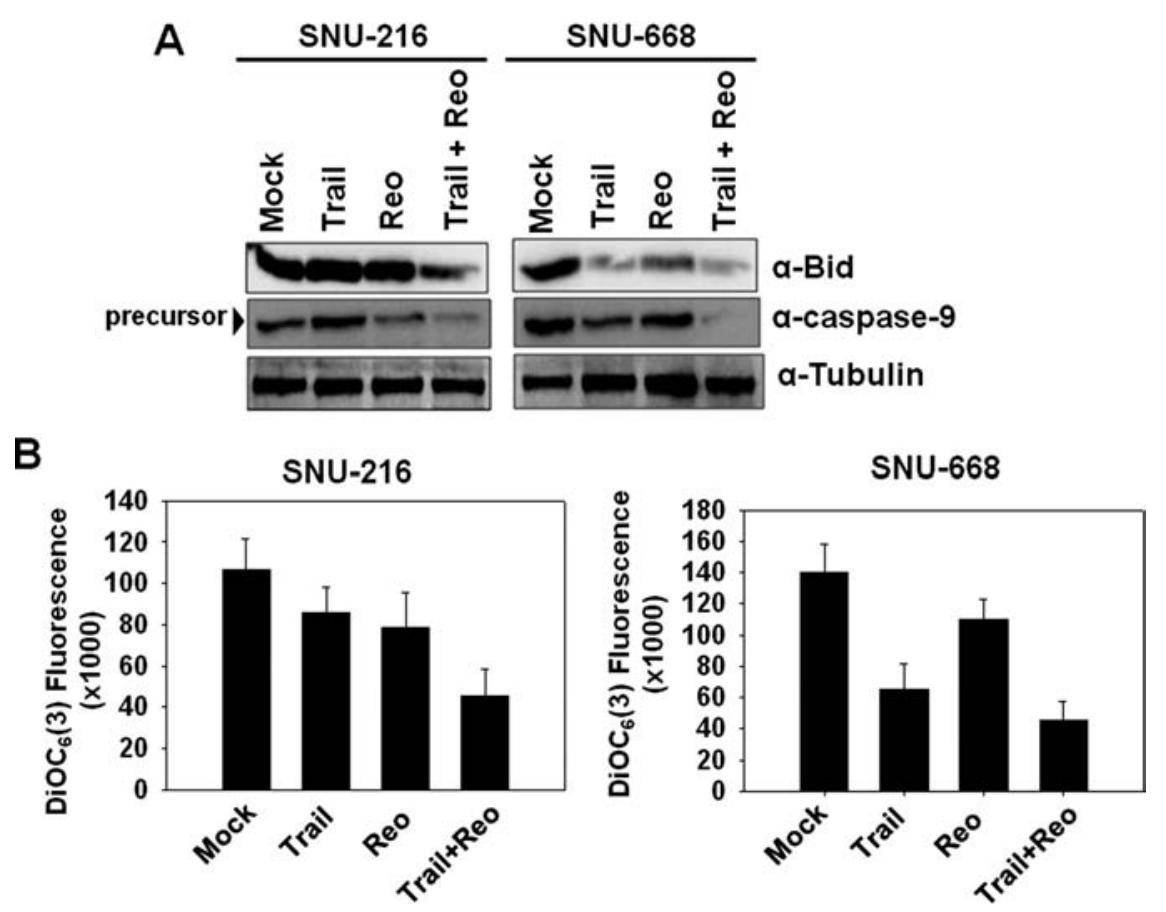

Figure 5. Co-treatment with reovirus and TRAIL reduces the mitochondrial membrane potential, leading to apoptotic cell death of SNU-216 cells. (A) SNU-216 cells were treated with TRAIL $(50 \mathrm{ng} / \mathrm{ml})$, reovirus $(\mathrm{MOI}=10)$ or reovirus plus TRAIL for $24 \mathrm{~h}$. Procaspase- 9 and Bid were detected by immunoblotting with the corresponding antibody. (B) SNU-216 cells were treated with TRAIL $(50 \mathrm{ng} / \mathrm{ml})$, reovirus $(\mathrm{MOI}=10)$ or reovirus plus TRAIL for $24 \mathrm{~h}$. The cells were then incubated with $40 \mathrm{nM} \mathrm{DiOC}_{6}(3)$ for 30 min and retention of $\operatorname{DiOC}_{6}(3)$ was measured using a fluorescence spectrometer. Results were averaged from three independent experiments and the error bars indicate standard deviations.

results in almost no Akt phosphorylation as seen in SNU-216 cells co-treated with reovirus and wortmannin (Fig. 4C). These combined results suggest that the co-treatment with reovirus and the PI3 kinase inhibitor, or reovirus and sh-Akt lentivirus efficiently induces apoptosis of SNU-216 gastric cancer cells by down-regulation of Akt activation.

Co-treatment with reovirus and TRAIL induces intrinsic apoptotic cell death in SNU-216 cells. Since we observed activation of caspase-8 during co-treatment of SNU-216 cells with reovirus and TRAIL, it is possible that Bid is cleaved by caspase- 8 , which would result in the generation of a 15 truncated Bid fragment that translocates to the mitochondria. Furthermore, it has been reported that truncated Bid is required for cytochrome $\mathrm{c}$ release from the mitochondria, which activates caspase- 9 and leads to mitochondria-mediated intrinsic apoptosis $(35,36)$. To test this hypothesis, we examined whether Bid or caspase-9 is cleaved during co-treatment of SNU-216 cells with reovirus and TRAIL. In these experiments, we found that Bid and caspase-9 were cleaved and the level of Bid or caspase-9 decreased in the combined treatment, whereas treatment with TRAIL or reovirus alone did not significantly induce Bid or caspase-9 cleavage (Fig. 5A). Using the fluorescent dye $\mathrm{DiOC}_{6}(3)$, we measured the mitochondrial membrane potential during co-treatment with reovirus and TRAIL in SNU-216 cells. From these experiments, we found that the combined treatment reduced the mitochondrial membrane potential by up to $~ 50 \%$ in SNU-216 cells, whereas treatment with TRAIL and reovirus alone reduced the mitochondrial membrane potential by 10 and $25 \%$, respectively, compared to the mock treatment. When we examined the mitochondrial membrane potential in TRAIL-sensitive SNU-668 cells, we observed a significant reduction in the mitochondrial membrane potential during TRAIL treatment alone $(\sim 50 \%)$ and the combined treatment $(\sim 70 \%)$ with TRAIL and reovirus when compared to the mock treatment. Treatment with reovirus alone marginally decreased the mitochondrial membrane potential in SNU-668 cells (Fig. 5B). Taken together, we propose that reovirus infection synergizes the effect of TRAIL on cleavage of Bid and caspase-9, resulting in a decrease in the mitochondrial membrane potential, which eventually leads to intrinsic apoptotic cell death.

\section{Discussion}

TRAIL-resistant SNU-216 gastric cancer cells displayed a much higher intrinsic Akt activity than TRAIL-sensitive SNU-668 cells, which suggests that active Akt levels are directly correlated with cell survival and resistance to TRAIL (9). The level of Akt thus determines the sensitivity of cells to TRAIL. This hypothesis was supported by previous studies where the inhibition of PI3 kinase/Akt signaling with wortmannin was found to cause TRAIL-induced apoptosis in LNCaP cells (a prostate cancer cell line) (7,37). In this study, we found that reovirus infection targets Akt protein, which leads to the down-regulation of Akt activation and enhances this effect in the presence of TRAIL. This observation was also supported by the result that wortmannin treatment or sh-Akt lentivirus infection accelerates reovirus-induced apoptosis in SNU-216 cells (Figs. 3C and 4B). Therefore, we suggest that down-regulation of Akt mediated by reovirus infection enhances the sensitivity of SNU-216 cells to TRAIL. 
We wondered how reovirus infection reduces Akt activation. To answer this question, we examined PTEN and Ras since it is known that loss of PTEN causes Akt activation (38) and Ras protein has a potential to activate PI-3 kinase/Akt signaling pathway (39). We found that reovirus infection down-regulates Ras activation but fail to activate PTEN. In addition, reovirus infection reduced the ERK activation but failed to down-regulate the activation of p38 MAPK in SNU216 cells, indicating that these events are specific features induced by reovirus infection (Fig. 2). Now, we are investigating more detailed mechanisms by which reovirus down-regulates the activation of Akt and Ras in SNU-216 gastric cancer cells.

The role of TRAIL receptors in TRAIL-mediated apoptosis have remained controversial because TRAIL receptors have been shown to increase in HEK293 cells during TRAIL treatment (40) but not in ZR75-1 (a breast cancer cell line) (24). Thus, when we examined the expression of DR4 and DR5 in SNU-216 cells treated with TRAIL, reovirus, or reovirus and TRAIL, we found no differential expression of DR4 and DR5 (data not shown). This result is also supported by another study where the expression level of DR4/DR5 and DcR2 were reported to be similar between uninfected SNU-216 and SNU-668 cells (9). Other studies have shown that alterations in TRAIL receptor expression do not fully explain TRAIL sensitivity $(41,42)$. These results also support the notion that TRAIL receptors do not play a critical role in our hypothesis; reovirus renders SNU-216 gastric cancer cells susceptible to TRAIL. Although we would not exclude a TRAIL receptor-mediated extrinsic death pathway, our results at least suggest that the reovirus may utilize the intrinsic death pathway because reovirus infection or co-treatment with reovirus and TRAIL reduces Akt activation, leading to the down-regulation of c-FLIP ${ }_{\mathrm{L}}$ (data not shown), which is involved in the inhibition of caspase- 8 activity (43). Furthermore, we observe a down-regulation of Bid level and activation of caspase-9 during co-treatment with reovirus and TRAIL, which led to great reduction in the mitochondrial membrane potential (Fig. 5). However, we have not observed any alteration in the level of Bax, Bim and Bcl-XL after reovirus infection (data not shown). The combined treatment with reovirus and TRAIL eventually resulted in caspase-8-initiated mitochondrial dysfunction, which ultimately led to apoptosis (Fig. 5). Based on the combined results of this study, we are currently investigating the mechanism by which the reovirus induces intrinsic apoptosis in SNU-216 cells in the presence of TRAIL.

\section{Acknowledgements}

This work was supported by the Bio-Scientific Research Grant funded by the Pusan National University (PNU-2008-101-214).

\section{References}

1. Ashkenazi A and Dixit VM: Death receptors: signaling and modulation. Science 281: 1305-1308, 1998.

2. Walczak H, Miller RE, Ariail K, Gliniak B, Griffith TS, Kubin M, Chin W, Jones J, Woodward A, Le T, Smith C, Smolak P, Goodwin RG, Rauch CT, Schuh JC and Lynch DH: Tumoricidal activity of tumor necrosis factor-related apoptosisinducing ligand in vivo. Nat Med 5: 157-163, 1999.
3. Wiley SR, Schooley K, Smolak PJ, Din WS, Huang CP, Nicholl JK, Sutherland GR, Smith TD, Rauch C, Smith CA, et al: Identification and characterization of a new member of the TNF family that induces apoptosis. Immunity 3: 673-682, 1995.

4. Bodmer JL, Holler N, Reynard S, Vinciguerra P, Schneider P, Juo P, Blenis J and Tschopp J: TRAIL receptor-2 signals apoptosis through FADD and caspase-8. Nat Cell Biol 2: 241243, 2000.

5. Muzio M, Stockwell BR, Stennicke HR, Salvesen GS and Dixit VM: An induced proximity model for caspase-8 activation. J Biol Chem 273: 2926-2930, 1998.

6. Boatright KM, Renatus M, Scott FL, Sperandio S, Shin H, Pedersen IM, Ricci JE, Edris WA, Sutherlin DP, Green DR and Salvesen GS: A unified model for apical caspase activation. Mol Cell 11: 529-541, 2003.

7. Nesterov A, Lu X, Johnson M, Miller GJ, Ivashchenko Y and Kraft AS: Elevated AKT activity protects the prostate cancer cell line LNCaP from TRAIL-induced apoptosis. J Biol Chem 276: 10767-10774, 2001

8. Asakuma J, Sumitomo M, Asano T, Asano T and Hayakawa M: Selective Akt inactivation and tumor necrosis actor-related apoptosis-inducing ligand sensitization of renal cancer cells by low concentrations of paclitaxel. Cancer Res 63: 1365-1370, 2003.

9. Nam SY, Jung GA, Hur GC, Chung HY, Kim WH, Seol DW and Lee BL: Upregulation of FLIP(S) by Akt, a possible inhibition mechanism of TRAIL-induced apoptosis in human gastric cancers. Cancer Sci 94: 1066-1073, 2003.

10. Zhang XD, Franco A, Myers K, Gray C, Nguyen T and Hersey P: Relation of TNF-related apoptosis-inducing ligand (TRAIL) receptor and FLICE-inhibitory protein expression to TRAIL-induced apoptosis of melanoma. Cancer Res 59: 2747-2753, 1999.

11. Mouzakiti A and Packham G: Regulation of tumour necrosis factor-related apoptosis-inducing ligand (TRAIL)-induced apoptosis in Burkitt's lymphoma cell lines. Br J Haematol 122: 61-69, 2003.

12. Kim M, Chung YH and Johnston RN: Reovirus and tumor oncolysis. J Microbiol 45: 187-192, 2007.

13. Duncan MR, Stanish SM and Cox DC: Differential sensitivity of normal and transformed human cells to reovirus infection. J Virol 28: 444-449, 1978

14. Hashiro G, Loh PC and Yau JT: The preferential cytotoxicity of reovirus for certain transformed cell lines. Arch Virol 54: 307-315, 1977

15. Strong JE, Coffey MC, Tang D, Sabinin P and Lee PW: The molecular basis of viral oncolysis: usurpation of the Ras signaling pathway by reovirus. EMBO J 17: 3351-3362, 1998.

16. Hirasawa K, Nishikawa SG, Norman KL, Coffey MC, Thompson BG, Yoon CS, Waisman DM and Lee PW: Systemic reovirus therapy of metastatic cancer in immune-competent mice. Cancer Res 63: 348-353, 2003.

17. Coffey MC, Strong JE, Forsyth PA and Lee PW: Reovirus therapy of tumors with activated Ras pathway. Science 282: 1332-1334, 1998

18. Norman KL and Lee PW: Not all viruses are bad guys: the case for reovirus in cancer therapy. Drug Discov Today 10: 847-855, 2005.

19. Park EH, Cho IR, Srisuttee R, Min HJ, Oh MJ, Jeong YJ, Jhun BH, Johnston RN, Lee SJ, Koh SS and Chung YH: CUG2, a novel oncogene confers reoviral replication through Ras and p38 signaling pathway. Cancer Gene Ther Jan.15,2010: doi: 10.1038/cgt.2009.83.

20. Park EH, Koh SS, Srisuttee R, Cho IR, Min HJ, Jhun BH, Lee YS, Jang KL, Kim CH, Johnston RN and Chung YH: Expression of HBX, an oncoprotein of hepatitis B virus, blocks reoviral oncolysis of hepatocellular carcinoma cells. Cancer Gene Ther 16: 453-461, 2009.

21. Cho IR, Koh SS, Min HJ, Park EH, Srisuttee R, Jhun BH, Jeong SH, Yoo YH, Youn HD, Johnston RN and Chung YH: Down-regulation of HIF-1alpha by oncolytic reovirus infection indepently of VHL and p53. Cancer Gene Ther Jan.15,2010: doi: $10.1038 /$ cgt.2009.84.

22. Stoeckel J and Hay JG: Drug evaluation: Reolysin-wild-type reovirus as a cancer therapeutic. Curr Opin Mol Ther 8: 249-260, 2006.

23. Janssen HL, Higuchi H, Abdulkarim A and Gores GJ: Hepatitis B virus enhances tumor necrosis factor-related apoptosisinducing ligand (TRAIL) cytotoxicity by increasing TRAILR1/death receptor 4 expression. J Hepatol 39: 414-420, 2003. 
24. Clarke P, Meintzer SM, Spalding AC, Johnson GL and Tyler KL: Caspase 8-dependent sensitization of cancer cells to TRAILinduced apoptosis following reovirus-infection. Oncogene 20: 6910-6919, 2001.

25. Parkin DM, Pisani P and Ferlay J: Estimates of the worldwide incidence of eighteen major cancers in 1985. Int J Cancer 54 594-606, 1993.

26. Lee YY, Kang SH, Seo JY, Jung CW, Lee KU, Choe KJ, Kim BK, Kim NK, Koeffler HP and Bang YJ: Alterations of p1 $6^{\mathrm{INK} 4 \mathrm{~A}}$ and $\mathrm{p} 15^{\mathrm{INK} 4 \mathrm{~B}}$ genes in gastric carcinomas. Cancer 80: 1889-1896, 1997.

27. Yokozaki H: Molecular characteristics of eight gastric cancer cell lines established in Japan. Pathol Int 50: 767-777, 2000.

28. Park JG, Yang HK, Kim WH, Chung JK, Kang MS, Lee JH, Oh JH, Park HS, Yeo KS, Kang SH, Song SY, Kang YK, Bang YJ, Kim YH and Kim JP: Establishment and characterization of human gastric carcinoma cell lines. Int J Cancer 70: 443-449, 1997.

29. Kim M, Egan C, Alain T, Urbanski SJ, Lee PW, Forsyth PA and Johnston RN: Acquired resistance to reoviral oncolysis in Ras-transformed fibrosarcoma cells. Oncogene 26: 4124-4134, 2007.

30. Norman KL, Hirasawa K, Yang AD, Shields MA and Lee PW: Reovirus oncolysis: the Ras/RalGEF/p38 pathway dictates host cell permissiveness to reovirus infection. Proc Natl Acad Sci USA 101: 11099-11104, 2004.

31. Follenzi A, Ailles LE, Bakovic S, Geuna M and Naldini L: Gene transfer by lentiviral vectors is limited by nuclear translocation and rescued by HIV-1 pol sequences. Nat Genet 25: 217-222, 2000.

32. Dull T, Zufferey R, Kelly M, Mandel RJ, Nguyen M, Trono D and Naldini L: A third-generation lentivirus vector with a conditional packaging system. J Virol 72: 8463-8471, 1998.

33. Srivastava RK, Sollott SJ, Khan L, Hansford R, Lakatta EG and Longo DL: Bcl-2 and Bcl-X(L) block thapsigargin-induced nitric oxide generation, c-Jun $\mathrm{NH}(2)$-terminal kinase activity and apoptosis. Mol Cell Biol 19: 5659-5674, 1999.
34. Alessi DR, Andjelkovic M, Caudwell B, Cron P, Morrice N, Cohen P and Hemmings BA: Mechanism of activation of protein kinase B by insulin and IGF-1. EMBO J 15: 6541-6551, 1996.

35. Cory S and Adams JM: The Bcl2 family: regulators of the cellular life-or-death switch. Nat Rev Cancer 2: 647-656, 2002.

36. Esposti MD: The roles of Bid. Apoptosis 7: 433-440, 2002.

37. Chen X, Thakkar H, Tyan F, Gim S, Robinson H, Lee C, Pandey SK, Nwokorie C, Onwudiwe N and Srivastava RK: Constitutively active Akt is an important regulator of TRAIL sensitivity in prostate cancer. Oncogene 20: 6073-6083, 2001.

38. Nielsen-Preiss SM, Silva SR and Gillette JM: Role of PTEN and Akt in the regulation of growth and apoptosis in human osteoblastic cells. J Cell Biochem 90: 964-975, 2003.

39. Zhang $\mathbf{J}$ and Lodish HF: Identification of K-ras as the major regulator for cytokine-dependent Akt activation in erythroid progenitors in vivo. Proc Natl Acad Sci USA 102: 14605-14610, 2005.

40. Clarke P, Meintzer SM, Gibson S, Widmann C, Garrington TP, Johnson GL and Tyler KL: Reovirus-induced apoptosis is mediated by TRAIL. J Virol 74: 8135-8139, 2000.

41. Keane MM, Ettenberg SA, Nau MM, Russell EK and Lipkowitz S: Chemotherapy augments TRAIL-induced apoptosis in breast cell lines. Cancer Res 59: 734-741, 1999.

42. Cuello M, Ettenberg SA, Clark AS, Keane MM, Posner RH, Nau MM, Dennis PA and Lipkowitz S: Down-regulation of the erbB-2 receptor by trastuzumab (herceptin) enhances tumor necrosis factor-related apoptosis-inducing ligand-mediated apoptosis in breast and ovarian cancer cell lines that overexpress erbB-2. Cancer Res 61: 4892-4900, 2001.

43. Clarke P and Tyler KL: Down-regulation of cFLIP following reovirus infection sensitizes human ovarian cancer cells to TRAIL-induced apoptosis. Apoptosis 12: 211-223, 2007. 